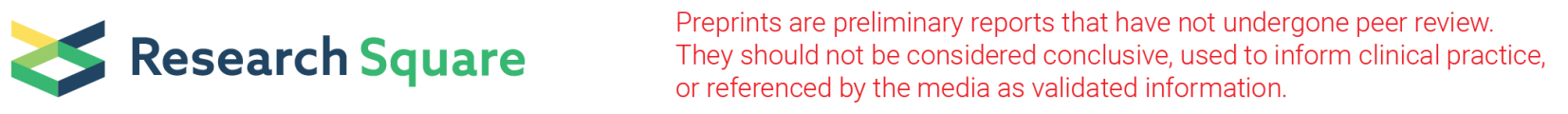

\title{
A pre-pandemic COVID-19 assessment of the costs of prevention and control interventions for healthcare associated infections in medical and surgical units in Québec
}

Eric Tchouaket ( $\square$ eric.tchouaket@uqo.ca )

Universite du Quebec en Outaouais https://orcid.org/0000-0002-4309-0478

Stephanie Robins

Université du Québec en Outaouais: Universite du Quebec en Outaouais

Sandra Boivin

Centre intégré de la santé et services sociaux des Laurentides

Drissa Sia

Universite du Quebec en Outaouais - Sciences infirmieres

Kelley Kilpatrick

McGill University Ingram School of Nursing

Bruno Dubreuil

Centre intégré de santé et services sociaux de Laval

Catherine Larouche

Centre universitaire de santé et services sociaux de Saguenay-Lac-St.Jean

Natasha Parisien

INSPQ: Institut national de sante publique du Quebec

Josiane Létourneau

Universite du Quebec en Outaouais - Sciences infirmieres

Research

Keywords: Healthcare associated infection, prevention and control, clinical best practice, cost, time-driven activity-based costing, time-motion study, COVID-19

Posted Date: June 14th, 2021

DOI: https://doi.org/10.21203/rs.3.rs-608797/v1

License: (c) This work is licensed under a Creative Commons Attribution 4.0 International License. Read Full License 


\section{Abstract \\ Background}

Healthcare-associated infections (HCAls) present a major public health problem that significantly affects patients, health care providers and the entire healthcare system. Infection prevention and control programs limit HCAls and are an indispensable component of patient and healthcare worker safety. The clinical best practices (CBPs) of handwashing, screening, hygiene and sanitation of surfaces and equipment, and basic and additional precautions are keystones of infection prevention and control (IPC). Systematic reviews of IPC economic evaluations report the lack of rigorous empirical evidence demonstrating the cost-benefit of IPC program in general, and point to the lack of assessment of the value of investing in CBPs more specifically.

\section{Objective}

This study aims to assess overall costs associated with each of the four CBPs.

\section{Methods}

Across two Quebec hospitals, 48 healthcare workers were observed for two hours each shift, for two consecutive weeks. A modified time-driven activitybased costing framework method was used to capture all human resources (time) and materials required (e.g. masks, cloths, disinfectants) for each clinical best practice. Using a hospital perspective with a time horizon of one year, median costs per CBP per hour, as well as the cost per action, were calculated and reported in 2018 Canadian dollars. Sensitivity analyses were performed.

\section{Results}

A total of 1831 actions were recorded. The median cost of hand hygiene $(\mathrm{N}=867)$ was 19.6 cents per action. For cleaning and disinfection of surfaces $(N=102)$, the cost was 21.4 cents per action, while cleaning of small equipment $(N=85)$ was 25.3 cents per action. Additional precautions median cost was $\$ 4.13$ per action. The donning or removing or personal protective equipment $(\mathrm{N}=720)$ cost was 75.9 cents per action. Finally, the total median costs for the five categories of clinical best practiced assessed were 27.2 cents per action.

\section{Conclusion}

The costs of clinical best practices were low, from 20 cents to $\$ 4.13$ per action. This study provides evidence based arguments with which to support the allocation of resources to infection prevention and control practices that directly affect the safety of patients, healthcare workers and the public. Further research of costing clinical best care practices is warranted.

\section{Introduction}

Healthcare-associated infections (HCAls) present a major public health problem that significantly affects patients, health care providers and the entire healthcare system. After necessary care in a clinic, hospital or long-term care facility, these infections can arise at surgical sites, following antibiotic therapy, or occur due to the use of devices such as ventilators, implants or catheters (1). Recent point prevalence surveys establish a range of HCAI rates across low, middle and higher income countries $(2,3)$. The highest rates of infection - up to $51.8 \%$ - occur in intensive care units, whereas overall hospital infection rates vary from $2.9 \%$ in six hospitals in Greece, to $9.2 \%$ in Australia to $10.4 \%$ in 25 hospitals in Canada and $14.3 \%$ in two hospitals in Ethiopia. Since most of these infections are considered preventable (4), they are seen as an indicator of the quality of patient care and safety. HCAls untowardly affect patients and their caregivers as they result in medical complications, high rates of morbidity and mortality, and reduced quality of life (5). They also burden healthcare systems in extra costs related to the prolonged length of stay or readmission of patients, patient's care-related expenses and costs involved in limiting further contagion $(6,7)$.

Accordingly, infection prevention and control (IPC) is an indispensable component of patient and healthcare worker safety. Essential IPC measures include four transverse clinical best care practices (CBPs) that apply across all care settings: a) hand hygiene; b) hygiene and sanitation including the cleaning and disinfecting of equipment and surfaces; c) application of basic and additional precautions (e.g., isolation, and donning and removing personal protective equipment) and; d) screening of carriers and patients who are at risk (8). These established components of IPC have been validated by extensive clinical practice and are incorporated in IPC guidelines set forth by the World Health Organization (WHO), Health Canada and the Canadian Patient Safety Institute $(9,10)$ as well as the US Centers for Disease Control and Prevention (CDC) $(9,11)$. In the current COVID-19 pandemic, these CBPs have proven vital in reducing the spread of infection in healthcare facilities $(12,13)$.

IPC programs that incorporate CBPs have been shown to be clinically, and from an institutional and government standpoint, cost effective (14, 15). Despite this, only a small proportion of most healthcare budgets are dedicated to public health programs which include IPC. In 2019 , the Canadian Institute for Health Information estimated Canada would spend \$264 billion Canadian dollars on health care, with a mere $5.4 \%$, or \$384 million, dedicated to public health programs (16). In Quebec's Economic Plan of 2018, less than $2 \%$ of the healthcare budget (representing $\$ 427$ million of 
\$21.8 billion) was destined for public health programs (17). A central hypothesis suggests this lack of investment is due to the dearth of rigorous empirical evidence demonstrating the cost-benefit of IPC programs. Systematic reviews of IPC economic evaluations report this gap in research in general, and point to the lack of assessment of the value of investing in CBPs more specifically $(6,14,18)$.

Comprehensive costing of activities involved in IPC must consider the costs of human resources and the costs of materials and products used. Analyzing human resource expenses can be undertaken using time-driven activity-based costing (TDABC), a process-oriented cost-accounting methodology increasingly used in healthcare to measure human resource costs $(19,20)$. TDABC functions by dynamically allocating expenses related to the consumption of resources across human-driven processes, with the purpose of summing them throughout the care delivery value chain (21). We previously developed a time and motion guide that captures resources consumed by healthcare worker (HCW) (22). The guide was specifically developed to assess the costs required to perform the CBPs presented above. It captures: (i) the time healthcare staff spend on: hand hygiene, cleaning and disinfecting healthcare equipment and the environment, donning and removing personal protective equipment (PPE), screening, basic practice and additional precautions (contact, droplet-contact or airborne), education, training and awareness campaigns; (ii) the materials used for these CBPs and; (iii) the products these CBPs require. Using micro-costing data collection that allows for cost estimates from the bottom up (23), the goal of this tool is to provide accurate IPC cost data to be used in economic healthcare evaluations and inform decision making (24).

As a first step in determining the cost-benefit of CBP IPC practices, this study aims to evaluate overall costs associated with each of the four CBPs by testing our time and motion guide in two healthcare facilities. To our knowledge, no study has undertaken an assessment of costs related to these four transverse CBPs, and none using a systematic and validated time and motion instrument. Moreover, no studies have been undertaken in Canada. Here we present the measurement of CBP costs in two healthcare facilities in the province of Québec, Canada. This evaluation will provide real cost data for healthcare decision makers regarding IPC implementation and optimal clinical practices. It will also provide researchers with insight about pre pandemic IPC healthcare costs, which will serve as a baseline from which to compare actual COVID-19 pandemic costs of IPC measures that were instituted in March of 2020.

Conceptual Framework and Research Questions

Our conceptual IPC framework is presented in Fig. 1, where the four CBPs are outlined. This framework was adapted from the theoretical framework of Resar and colleagues (8), and previously used by our team (22). In this study, we aim to answer the following questions in the context of an acute care setting: what are the human and material resource costs of: 1) hand hygiene, 2) hygiene and sanitation practices, and disinfection of equipment, 3 ) basic practices and additional precautions (donning and removing personal protective equipment or PPE) and 4) screening?

\section{Methods Design}

A prospective observational study was used. Data were collected in the pre-COVID-19 period between November 28th and December 15 th, 2018 from one hospital in the Saguenay-Lac-Saint-Jean region and one hospital in the Laurentian region of Québec, Canada. We chose a university and nonuniversity based integrated health and social services centre.

\section{Participants and procedures}

HCWs were selected from acute-care wards of medicine and surgery as these wards see and treat the highest number of hospitalized patients. In each hospital, the selected staff included 6 nurses, 6 auxiliary nurses, 6 orderlies and 6 hygiene and sanitation workers providing a final sample of 48 (24 from each site). Prospective participants were approached by the research team and unit managers, or at information sessions, and had the study explained to them. If they agreed, participants provided written informed consent. Each participant was directly observed by a nurse researcher over a two-hour period during their regularly scheduled work shift (day or evening), for two consecutive weeks. The time spent in relation to each CBP (hand hygiene; hygiene and sanitation including the cleaning and disinfecting of equipment and surfaces; application of basic and additional precautions including donning and removing of PPE and screening of carriers and patients who are at risk), along with all materials and product used were measured. Ethical approval was obtained from the sites where recruitment took place (study \# MP-28-2018-002).

\section{Time and motion guide}

The development of the IPC time and motion guide used in this study has been previously described (22). It was based on an algorithm developed by our team, and published. Validated by Delphi review, the guide contains eight dimensions of human and material resource assessment. These include: Identification [83 items]; Personnel [5 items]; Additional Precautions [1 item]; Hand Hygiene [2 items]; Personal Protective Equipment [14 items]; Screening [4 items]; Cleaning and Disinfection of Patient Care Equipment [33 items]; and Hygiene and Sanitation [24 items]. Observers follow one HCW and, using an online version of the guide, systematically record time of actions as triggered from the beginning to the end of the action, using chronometric measurement. The observer inputs products (e.g. hand soap or hydro-alcoholic solution) and disposable and reusable materials (e.g. gowns, wipes, bedpans) used during these procedures. We followed the suggested time and motion definitions set out by Lopetegui et al. (25). As these authors suggest, for time measurement, the guide requires an 'external observer capturing data continuously' and is characterized by a 'milestone' study schema, where all work time in relation to a particular action (motion) is measured in seconds. This process allows for the collection of valid and objective real-time measurements (Supplementary files 1 and 2). 


\section{The Procedure of Data Analysis for Costing}

We used a hospital perspective with a time horizon of one year as reported in 2018 . All costs were actualized to 2021 using a discount rate of $3 \%$, $5 \%$ and $8 \%$ and reported in Canadian dollars (\$CAD). All human resource costs (salaries) were based on standardized government salaries for each HCW (26). Costs of all supplies were obtained from a standardized provincial public healthcare pricelist for hospitals 2017-2018 (27). Our costing procedure (see Fig. 2) is similar to that described by da Silva Etges et al.(28), who propose a modified 8-step TDABC micro-costing framework originally set out by Keel and colleagues $(20,28)$. These authors break down healthcare-based costing into: 1$)$ defining the study question or process to be assessed; 2 ) mapping the process; 3 ) identifying the resource groups (structure and personnel) used in each activity; 4) estimating the total cost of each resource group; 5) estimating capacity per resource and the cost capacity rate; 6 ) analysing the time required for each activity used during the process; 7 ) calculating the total cost of patient care and; 8) performing cost analytics (benchmarking, costing per phase of care etc.). We followed the above in our costing process, with several small adaptations. First, we defined our process not as care delivery as seen from the patient perspective, but rather as those events undertaken by HCWs in relation to CBPs performed across a set period of time. Second, our resource groups did not include overhead costs, all of our costs were operating expenses (here defined as human, material and product resources used in IPC). Finally, we simplified our capacity cost rate (29) for human resource costing: our numerator was the worker's salary and their benefits, the denominator was an hour of time.

\section{Costing}

To obtain human resource costs, the number of actions undertaken by HCWs was multiplied by the average time (in seconds) required for that action and by the average salary (including benefits) for that HCW category. To calculate material costs, the total number of disposable items (e.g. microfiber cloths, masks) that were used was multiplied by the price per item. For materials that were reusable, the total number of items used was multiplied by the depreciation rate of that item (i.e. number of possible uses). Products were costed by multiplying the number of actions by the cost per volume of product used for each action (see Table 1). These calculations provided a total cost over the two-hour observation period. We also report cost per hour. IBM SPSS Statistics for Mac, Version 26.0 (Armonk, NY: IBM Corp) was used for all calculations of cost.

Table 1.

\section{Cost Calculation of Clinical Best Practice Actions}

\begin{tabular}{|lll|}
\hline Resource Cost & Unit & Multiplied by \\
\hline Human & All time in seconds converted to hourly scale & Hourly wage including benefits \\
\hline Material: disposable & Quantity used & Price per item \\
\hline Material: reusable & Quantity used & \% depreciation (number of times used) \\
\hline Product (e.g. soap) & Millilitres (mls) of product & Price per item \\
\hline Product (e.g. disinfectant) & Millilitres (mls) of diluent & Price per ml* \\
\hline Total Costs of CBPs & All human resource+ material + product costs & \\
\hline$*$ Costs will be multiplied by proportion of total solution used or discarded & \\
\hline
\end{tabular}

\section{Sensitivity analyses}

For all human resources, sensitivity analyses were performed to account for the range of salaries (minimum to maximum) of professionals, depending on the salary scale for each profession. Sensitivity analyses were also performed for time spent performing each CBP using the minimum and maximum limit respectively. For products where the volume varies from a standard quantity depending on the user (e.g. millilitres of soap for hand washing), sensitivity analyses were performed by using a range of volumes. Using Monte Carlo simulation, we varied each cost over 1,000 iterations, to obtain $2.5 \%$ and $97.5 \%$ percentiles and estimate the $95 \%$ confidence interval. Microsoft excel was used to perform the sensitivity analyses

\section{Comparison analyses}

We compared time spent across category (e.g. human resource or product) within each CBP using a 5\% threshold and non-parametric tests (MannWhitney Wilcoxon, Kruskal Wallis) (30).

\section{Results}

Times and actions used for clinical best practices

All CBP times are described in Table 2. 
Table 2

Number of actions and time used for clinical best practices

\begin{tabular}{|c|c|c|c|c|c|c|c|c|c|c|c|c|}
\hline \multirow{2}{*}{$\begin{array}{l}\text { Clinical best } \\
\text { practice }\end{array}$} & \multicolumn{2}{|c|}{ Actions } & \multirow{2}{*}{$\begin{array}{l}\text { Median } \\
\text { Time } \\
\text { (seconds) }\end{array}$} & \multicolumn{2}{|c|}{ Percentiles } & \multirow{2}{*}{$\begin{array}{l}\text { Mean } \\
\text { Time } \\
\text { (seconds) }\end{array}$} & \multirow{2}{*}{$\begin{array}{l}\text { S.D. } \\
\text { (seconds) }\end{array}$} & \multirow{2}{*}{$\begin{array}{l}\text { Minimum } \\
\text { Time } \\
\text { (seconds) }\end{array}$} & \multirow{2}{*}{$\begin{array}{l}\text { Maximum } \\
\text { Time } \\
\text { (seconds) }\end{array}$} & \multicolumn{3}{|c|}{ Kruskal Wallis } \\
\hline & $\mathbf{N}$ & $\%$ & & 2.5 & 97.5 & & & & & $x^{2}$ & df & $\mathbf{P}$ \\
\hline Hand hygiene & 867 & 100 & 14.32 & 4.01 & 35.69 & 15.59 & 8.33 & 1.40 & 109.85 & & & \\
\hline \multicolumn{13}{|l|}{$\begin{array}{l}\text { Healthcare } \\
\text { personnel }\end{array}$} \\
\hline $\begin{array}{l}\text { Nurse: bachelor } \\
\text { trained }\end{array}$ & 21 & 2.4 & 14.94 & 11.04 & 27.85 & 16.88 & 5.09 & 11.04 & 27.85 & \multirow[t]{5}{*}{21.27} & \multirow[t]{5}{*}{4} & \multirow[t]{5}{*}{0.000} \\
\hline $\begin{array}{l}\text { Nurse: college } \\
\text { trained }\end{array}$ & 125 & 14.4 & 16.04 & 4.20 & 40.04 & 17.80 & 11.45 & 2.47 & 109.85 & & & \\
\hline Auxiliary nurse & 244 & 28.1 & 13.52 & 3.20 & 38.50 & 15.19 & 8.74 & 2.42 & 49.32 & & & \\
\hline Orderly & 347 & 40 & 14.55 & 5.59 & 35.75 & 15.78 & 7.17 & 2.68 & 46.68 & & & \\
\hline $\begin{array}{l}\text { Hygiene and } \\
\text { sanitation staff }\end{array}$ & 130 & 15 & 12.41 & 2.98 & 31.03 & 13.51 & 6.64 & 1.40 & 41.47 & & & \\
\hline \multicolumn{10}{|l|}{ Moment } & \multirow[t]{6}{*}{36.86} & \multirow[t]{6}{*}{4} & \multirow[t]{6}{*}{0.000} \\
\hline $\begin{array}{l}\text { Before patient } \\
\text { contact }\end{array}$ & 193 & 22.3 & 14.08 & 5.08 & 34.34 & 14.98 & 7.00 & 2.55 & 40.17 & & & \\
\hline $\begin{array}{l}\text { Before aseptic } \\
\text { procedure }\end{array}$ & 17 & 2.0 & 21.47 & 5.24 & 32.50 & 20.09 & 7.13 & 5.24 & 32.50 & & & \\
\hline $\begin{array}{l}\text { After patient } \\
\text { contact }\end{array}$ & 601 & 69.3 & 14.07 & 3.44 & 32.72 & 15.02 & 7.27 & 1.40 & 49.32 & & & \\
\hline $\begin{array}{l}\text { After body fluid } \\
\text { exposure }\end{array}$ & 37 & 4.3 & 14.90 & 3.99 & 109.85 & 19.94 & 18.58 & 3.99 & 109.85 & & & \\
\hline Other & 19 & 2.2 & 24.11 & 14.63 & 43.42 & 27.35 & 9.74 & 14.63 & 43.42 & & & \\
\hline \multicolumn{10}{|l|}{ Products used } & \multirow[t]{5}{*}{54.18} & \multirow[t]{5}{*}{2} & \multirow[t]{5}{*}{0.000} \\
\hline Soap and water & 71 & 8.2 & 22.16 & 8.48 & 44.07 & 23.46 & 9.97 & 8.22 & 46.68 & & & \\
\hline $\begin{array}{l}\text { Hydroalcoholic } \\
\text { solution wall }\end{array}$ & 761 & 87.8 & 13.95 & 4.02 & 32.74 & 14.87 & 7.75 & 1.40 & 109.85 & & & \\
\hline $\begin{array}{l}\text { Hydroalcoholic } \\
\text { solution table } \\
(500 \mathrm{ml})\end{array}$ & 32 & 3.7 & 13.83 & 2.55 & 29.43 & 13.79 & 7.73 & 2.55 & 29.43 & & & \\
\hline $\begin{array}{l}\text { Hydroalcoholic } \\
\text { solution pocket } \\
\text { size }(45-50 \mathrm{ml})\end{array}$ & 3 & 0.3 & 27.77 & 27.24 & 36.39 & 30.47 & 5.13 & 27.24 & 36.39 & & & \\
\hline $\begin{array}{l}\text { Hygiene and } \\
\text { sanitation of } \\
\text { surfaces }\end{array}$ & 102 & 100 & 541.53 & 42.91 & 1840.23 & 598.59 & 486.14 & 35.40 & 3411.55 & & & \\
\hline \multicolumn{13}{|l|}{$\begin{array}{l}\text { Healthcare } \\
\text { personnel }\end{array}$} \\
\hline $\begin{array}{l}\text { Hygiene and } \\
\text { sanitation staff }\end{array}$ & 102 & 100 & 541.53 & 42.91 & 1840.23 & 598.59 & 486.14 & 35.40 & 3411.55 & & \multicolumn{2}{|l|}{$\mathrm{n} / \mathrm{a}$} \\
\hline \multicolumn{10}{|l|}{ Kind of cleaning } & \multirow[t]{5}{*}{32.45} & \multirow[t]{5}{*}{3} & \multirow[t]{5}{*}{0.000} \\
\hline Daily regular & 58 & 56.9 & 333.57 & 41.45 & 1140.61 & 432.70 & 300.09 & 35.40 & 1222.16 & & & \\
\hline $\begin{array}{l}\text { Daily additional } \\
\text { precautions }\end{array}$ & 13 & 12.7 & 335.88 & 51.42 & 701.99 & 391.19 & 181.56 & 51.42 & 701.99 & & & \\
\hline Terminal & 3 & 2.9 & 738.59 & 35.83 & 3411.55 & 1395.32 & 1781.11 & 35.83 & 3411.55 & & & \\
\hline $\begin{array}{l}\text { Terminal } \\
\text { discharge / } \\
\text { transfer }\end{array}$ & 28 & 27.5 & 872.53 & 406.01 & 1940.97 & 953.11 & 404.48 & 406.01 & 1940.97 & & & \\
\hline \multicolumn{13}{|l|}{ Materials Used* } \\
\hline Reusable cloths & 6 & 5.9 & 635.89 & 450.69 & 1492.80 & 752.97 & 390.23 & 450.69 & 1492.80 & $\mathrm{n} / \mathrm{a}$ & & \\
\hline
\end{tabular}




\begin{tabular}{|c|c|c|c|c|c|c|c|c|c|c|c|c|}
\hline \multirow{2}{*}{$\begin{array}{l}\text { Clinical best } \\
\text { practice }\end{array}$} & \multicolumn{2}{|c|}{ Actions } & \multirow{2}{*}{$\begin{array}{l}\text { Median } \\
\text { Time } \\
\text { (seconds) }\end{array}$} & \multicolumn{2}{|c|}{ Percentiles } & \multirow{2}{*}{$\begin{array}{l}\text { Mean } \\
\text { Time } \\
\text { (seconds) }\end{array}$} & \multirow{2}{*}{$\begin{array}{l}\text { S.D. } \\
\text { (seconds) }\end{array}$} & \multirow{2}{*}{$\begin{array}{l}\text { Minimum } \\
\text { Time } \\
\text { (seconds) }\end{array}$} & \multirow{2}{*}{$\begin{array}{l}\text { Maximum } \\
\text { Time } \\
\text { (seconds) }\end{array}$} & \multicolumn{3}{|c|}{ Kruskal Wallis } \\
\hline & $\mathbf{N}$ & $\%$ & & 2.5 & 97.5 & & & & & $x^{2}$ & df & $\mathbf{P}$ \\
\hline $\begin{array}{l}\text { Microfibre- } \\
\text { reuseable }\end{array}$ & 97 & 95.1 & 554.86 & 41.37 & 1862.13 & 611.70 & 493.57 & 35.40 & 3411.55 & & & \\
\hline $\begin{array}{l}\text { Disinfectant } \\
\text { disposable wipes }\end{array}$ & 1 & 1.0 & 565.46 & 565.46 & 565.46 & 565.46 & - & 565.46 & 565.46 & & & \\
\hline Alcohol swabs & 0 & 0 & - & - & - & - & - & - & - & & & \\
\hline Floor buffers & 19 & 18.2 & 596.77 & 320.35 & 1531.68 & 715.17 & 350.11 & 320.35 & 1531.68 & & & \\
\hline Mops & 1 & 1.0 & 58.61 & 58.61 & 58.61 & 58.61 & - & 58.61 & 58.61 & & & \\
\hline Toilette brush & 1 & 1.0 & 151.90 & 151.90 & 151.90 & 151.90 & - & 151.90 & 151.90 & & & \\
\hline \multicolumn{13}{|l|}{$\begin{array}{l}\text { *NB the number } \\
\text { of materials } \\
\text { surpasses the } \\
\text { total number of } \\
\text { actions as } \\
\text { actions can use } \\
\text { more than one } \\
\text { material/product. }\end{array}$} \\
\hline $\begin{array}{l}\text { Cleaning of } \\
\text { small equipment }\end{array}$ & 85 & 100 & 31.29 & 10.81 & 91.29 & 34.81 & 18.61 & 8.72 & 94.93 & & & \\
\hline \multicolumn{13}{|l|}{$\begin{array}{l}\text { Healthcare } \\
\text { personnel }\end{array}$} \\
\hline $\begin{array}{l}\text { Nurse: bachelor } \\
\text { trained }\end{array}$ & 2 & 2.4 & 21.83 & 21.09 & 22.57 & 21.83 & 1.05 & 21.09 & 22.57 & 4.15 & 4 & 0.386 \\
\hline $\begin{array}{l}\text { Nurse: college } \\
\text { trained }\end{array}$ & 23 & 27.1 & 29.99 & 11.70 & 57.24 & 33.52 & 12.66 & 11.70 & 57.24 & & & \\
\hline Auxiliary nurse & 38 & 44.7 & 28.03 & 8.72 & 94.93 & 35.28 & 23.69 & 8.72 & 94.93 & & & \\
\hline Orderly & 12 & 14.1 & 36.09 & 14.00 & 85.65 & 39.36 & 17.72 & 14.00 & 85.65 & & & \\
\hline $\begin{array}{l}\text { Hygiene and } \\
\text { sanitation staff }\end{array}$ & 10 & 11.8 & 35.97 & 16.59 & 42.62 & 33.14 & 8.64 & 16.59 & 42.62 & & & \\
\hline \multicolumn{13}{|l|}{$\begin{array}{l}\text { Type of small } \\
\text { equipment }\end{array}$} \\
\hline $\begin{array}{l}\text { Multifunction } \\
\text { vital sign monitor }\end{array}$ & 29 & 34.1 & 39.65 & 11.46 & 94.92 & 43.33 & 20.79 & 11.46 & 94.92 & & & \\
\hline $\begin{array}{l}\text { Blood glucose } \\
\text { monitor }\end{array}$ & 18 & 21.2 & 20.74 & 8.72 & 51.71 & 21.60 & 10.00 & 8.72 & 51.71 & & & \\
\hline $\begin{array}{l}\text { Chariot and mop } \\
\text { handle }\end{array}$ & 10 & 11.8 & 32.19 & 16.59 & 42.62 & 30.53 & 9.03 & 16.59 & 42.62 & & & \\
\hline $\begin{array}{l}\text { Positive } \\
\text { displacement } \\
\text { pump }\end{array}$ & 4 & 4.7 & 58.28 & 40.87 & 83.23 & 60.17 & 17.44 & 40.87 & 83.23 & & & \\
\hline Pulse oximeter & 4 & 4.7 & 37.00 & 21.08 & 65.02 & 40.02 & 18.70 & 21.08 & 65.02 & & & \\
\hline $\begin{array}{l}\text { Portable bladder } \\
\text { scanner }\end{array}$ & 3 & 3.5 & 21.88 & 20.79 & 22.98 & 21.88 & 1.09 & 20.79 & 22.98 & & & \\
\hline Wheelchair & 3 & 3.5 & 22.11 & 14.00 & 34.74 & 23.62 & 10.45 & 14.00 & 34.74 & & & \\
\hline Commode chair & 3 & 3.5 & 32.50 & 25.72 & 44.55 & 34.26 & 9.54 & 25.72 & 44.55 & & & \\
\hline Walker & 2 & 2.4 & 35.98 & 20.15 & 51.82 & 35.98 & 22.39 & 20.15 & 51.82 & & & \\
\hline Scissors & 3 & 3.5 & 15.37 & 11.70 & 29.17 & 18.74 & 9.21 & 11.70 & 29.17 & & & \\
\hline Emesis bowl & 3 & 3.5 & 37.45 & 29.89 & 41.98 & 36.44 & 6.11 & 29.89 & 41.98 & & & \\
\hline Thermometer & 2 & 2.4 & 79.97 & 65.02 & 94.93 & 79.97 & 21.15 & 65.02 & 94.93 & & & \\
\hline Stretcher & 1 & 1.2 & 85.65 & 85.65 & 85.65 & 85.65 & - & 85.65 & 85.65 & & & \\
\hline
\end{tabular}




\begin{tabular}{|c|c|c|c|c|c|c|c|c|c|c|c|c|}
\hline \multirow{2}{*}{$\begin{array}{l}\text { Clinical best } \\
\text { practice }\end{array}$} & \multicolumn{2}{|c|}{ Actions } & \multirow{2}{*}{$\begin{array}{l}\text { Median } \\
\text { Time } \\
\text { (seconds) }\end{array}$} & \multicolumn{2}{|c|}{ Percentiles } & \multirow{2}{*}{$\begin{array}{l}\text { Mean } \\
\text { Time } \\
\text { (seconds) }\end{array}$} & \multirow{2}{*}{$\begin{array}{l}\text { S.D. } \\
\text { (seconds) }\end{array}$} & \multirow{2}{*}{$\begin{array}{l}\text { Minimum } \\
\text { Time } \\
\text { (seconds) }\end{array}$} & \multirow{2}{*}{$\begin{array}{l}\text { Maximum } \\
\text { Time } \\
\text { (seconds) }\end{array}$} & \multicolumn{3}{|c|}{ Kruskal Wallis } \\
\hline & $\mathbf{N}$ & $\%$ & & 2.5 & 97.5 & & & & & $x^{2}$ & df & $P$ \\
\hline $\begin{array}{l}\text { Laundry bag } \\
\text { support }\end{array}$ & 1 & 1.2 & 44.71 & 44.71 & 44.71 & 44.71 & - & 44.71 & 44.71 & & & \\
\hline Bassin & 1 & 1.2 & 35.70 & 35.70 & 35.70 & 35.70 & - & 35.70 & 35.70 & & & \\
\hline Scale & 1 & 1.2 & 29.30 & 29.30 & 29.30 & 29.30 & - & 29.30 & 29.30 & & & \\
\hline Doppler & 1 & 1.2 & 37.29 & 37.29 & 37.29 & 37.29 & - & 37.29 & 37.29 & & & \\
\hline Table & 1 & 1.2 & 33.28 & 33.28 & 33.28 & 33.28 & - & 33.28 & 33.28 & & & \\
\hline Comb & 1 & 1.2 & 22.38 & 22.38 & 22.38 & 22.38 & - & 22.38 & 22.38 & & & \\
\hline Other & 18 & 21.2 & 33.18 & 11.70 & 44.71 & 31.77 & 9.19 & 11.70 & 44.71 & & & \\
\hline $\begin{array}{l}\text { Additional } \\
\text { precautions }\end{array}$ & 57 & 100 & 274.30 & 16.10 & 2668.96 & 384.95 & 511.66 & 15.53 & 3445.84 & & & \\
\hline $\begin{array}{l}\text { Healthcare } \\
\text { personnel }\end{array}$ & & & & & & & & & & 23.09 & 3 & 0.000 \\
\hline $\begin{array}{l}\text { Nurse: bachelor } \\
\text { trained }\end{array}$ & 5 & 8.8 & 169.05 & 32.30 & 205.35 & 138.92 & 77.37 & 32.30 & 205.35 & & & \\
\hline $\begin{array}{l}\text { Nurse: college } \\
\text { trained }\end{array}$ & 0 & 0 & - & - & - & - & - & - & - & & & \\
\hline Auxiliary nurse & 17 & 29.8 & 199.80 & 15.54 & 579.91 & 247.79 & 160.89 & 15.53 & 579.91 & & & \\
\hline Orderly & 16 & 28.1 & 117.58 & 16.80 & 786.47 & 197.50 & 213.04 & 16.80 & 786.47 & & & \\
\hline $\begin{array}{l}\text { Hygiene and } \\
\text { sanitation staff }\end{array}$ & 19 & 33.3 & 542.80 & 89.83 & 3445.84 & 730.27 & 748.74 & 89.83 & 3445.84 & & & \\
\hline \multicolumn{13}{|l|}{$\begin{array}{l}\text { Kind of } \\
\text { additional } \\
\text { precaution }\end{array}$} \\
\hline Contact & 42 & 73.7 & 252.92 & 16.66 & 3316.36 & 427.71 & 586.03 & 15.54 & 3445.84 & 4.96 & 3 & 0.000 \\
\hline Contact- droplet & 12 & 21.1 & 340.94 & 89.83 & 463.83 & 313.38 & 114.26 & 89.83 & 463.83 & & & \\
\hline Droplet & 2 & 3.5 & 100.45 & 84.85 & 116.06 & 100.45 & 22.07 & 84.85 & 116.06 & & & \\
\hline $\begin{array}{l}\text { Protection } \\
\text { (inversed) }\end{array}$ & 1 & 1.8 & 16.80 & 16.80 & 16.80 & 16.80 & - & 16.80 & 16.80 & & & \\
\hline Airborne & - & - & - & - & - & - & - & - & - & & & \\
\hline Airborne-contact & - & - & - & - & - & - & - & - & - & & & \\
\hline \multirow{2}{*}{$\begin{array}{l}\text { Personal } \\
\text { Protective } \\
\text { Equipment (PPE) }\end{array}$} & 720 & 100 & 11.58 & 2.78 & 48.11 & 13.95 & 10.59 & 0.26 & 94.38 & & & \\
\hline & & & & & & & & & & 11.09 & 4 & 0.026 \\
\hline \multicolumn{13}{|l|}{$\begin{array}{l}\text { Healthcare } \\
\text { personnnel }\end{array}$} \\
\hline $\begin{array}{l}\text { Nurse: bachelor } \\
\text { trained }\end{array}$ & 12 & 1.7 & 16.52 & 9.90 & 58.68 & 24.57 & 15.22 & 9.90 & 58.68 & & & \\
\hline $\begin{array}{l}\text { Nurse: college } \\
\text { trained }\end{array}$ & 37 & 5.1 & 11.11 & 3.85 & 28.26 & 12.13 & 5.91 & 3.85 & 28.26 & & & \\
\hline Auxiliary nurse & 125 & 17.4 & 11.75 & 1.93 & 40.31 & 13.94 & 9.89 & 1.60 & 75.95 & & & \\
\hline Orderly & 243 & 33.8 & 11.50 & 4.54 & 39.01 & 13.24 & 7.89 & 3.15 & 65.49 & & & \\
\hline $\begin{array}{l}\text { Hygiene and } \\
\text { sanitation }\end{array}$ & 303 & 42.1 & 11.74 & 2.02 & 64.50 & 14.33 & 12.62 & 0.26 & 94.38 & & & \\
\hline \multicolumn{13}{|l|}{ PPE used } \\
\hline Disposable gown & 160 & 22.2 & 13.24 & 5.89 & 75.37 & 18.73 & 15.74 & 1.69 & 94.38 & $\mathrm{n} / \mathrm{a}$ & & \\
\hline
\end{tabular}




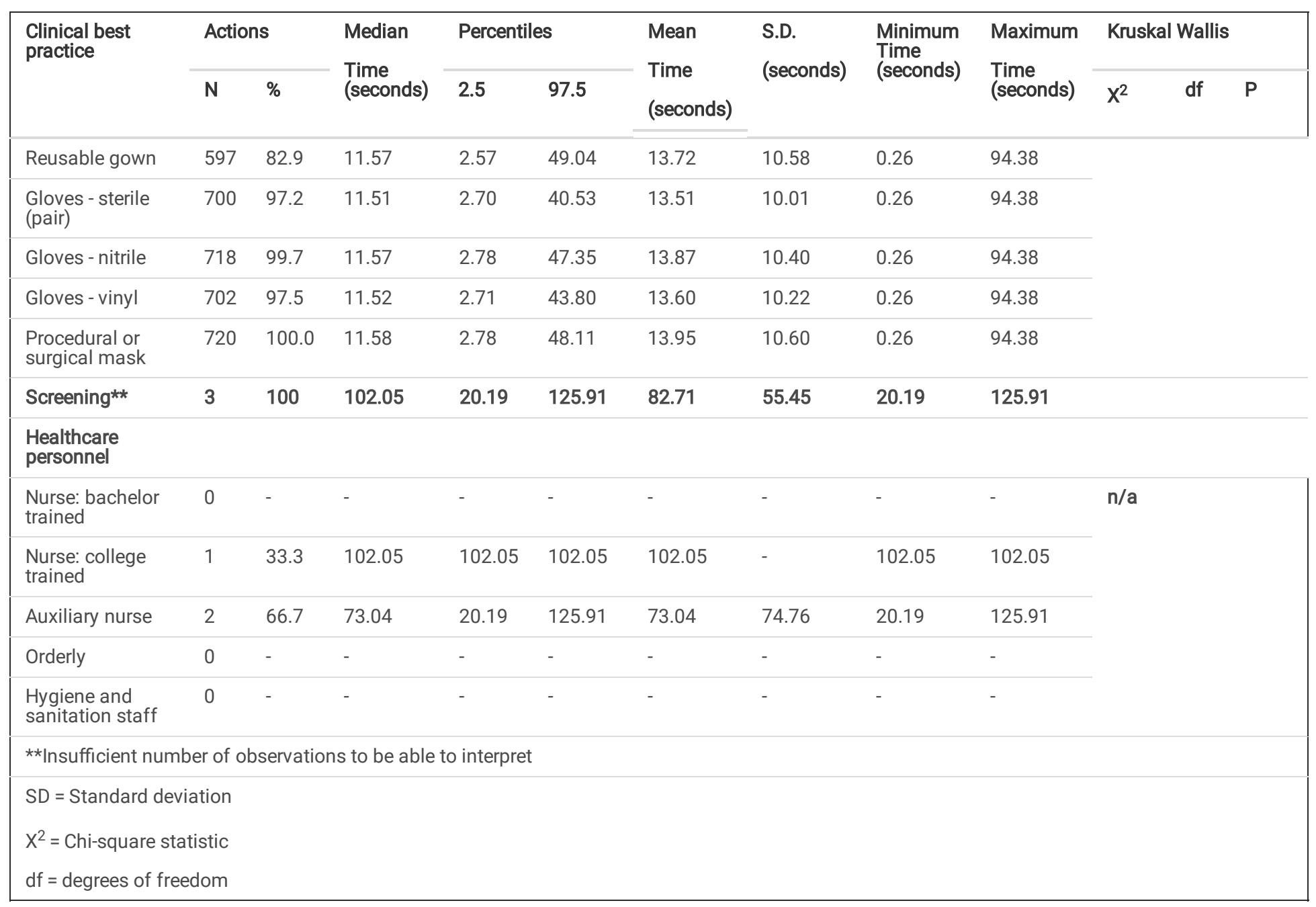

\section{Hand hygiene}

A total of 867 hand hygiene actions took place; median hand washing time was 14.32 seconds (range 1.40-109.85) across all staff (nurses, orderlies and hygiene and sanitation staff). There was a significant difference $X^{2}=21.27 p=0.000$ between groups. Moreover, the moment when hands were washed differed significantly $X^{2}=36.86 p=0.000$ and was highest before an aseptic procedure at 21.47 seconds.

With regards to different products used, pocket size hydroalcoholic solution had the longest median hand washing time at 27.77 seconds, followed by soap and water at 22.16 seconds, then table sized hydroalcoholic solution $500 \mathrm{ml}$, and wall at 13.95 and 13.83 seconds respectively. Time across these products differed significantly $\mathrm{X}^{2}=54.18 p=0.000$.

\section{Hygiene and sanitation of surfaces and cleaning and disinfecting of equipment}

A total of 102 actions took place to clean surfaces, exclusively by hygiene and sanitation staff. The median time spent cleaning was 541.63 seconds (or 9 minutes and 2 seconds) range 35.40 seconds to 3411.55 seconds ( 56 minutes and 52 seconds). The most time was dedicated to terminal cleaning (cleaning following patient transfer or discharge) with a median of 872.53 seconds (or 14 minutes and 33 seconds). The kind of cleaning differed $X^{2}=$ $32.45 p=0.000$, significantly by category.

There were 85 actions related to the cleaning of small equipment with the median time being just under half a minute at 31.29 seconds (range $8.72-$ 94.93). There was no significant difference between personnel.

\section{Personal protective equipment (PPE)}

Furthermore, a total of 720 actions were related to the donning or removing of personal protective equipment (PPE). Median time for PPE was 11.58 seconds (range 1.0-94.38). Bachelor trained nurses had the highest median at 16.52 seconds per action compared to an average of 11 seconds for all other personnel, who differed significantly $\mathrm{X}^{2}=23.09 p=0.026$.

\section{Additional precautions}

For the CBP of additional precautions 57 actions were recorded, with a median time of 274.30 seconds (4 minutes and 34 seconds), (range $15.53-$ 3445.84 seconds or 57 minutes and 26 seconds). There was a significant difference between personnel $X^{2}=23.09 p=0.000$. Contact - droplet 
precautions were the kind of precaution with the highest median at 340.94 seconds. There were no observations of airborne or airborne-contact precautions during the study.

\section{Screening}

There were insufficient recordings of screening procedures $(\mathrm{N}=3)$ to describe them.

\section{CBP Costs}

A summary of CBP costs are described in Table 3.

Table 3

Costs of clinical best practices over two hour observation period

\begin{tabular}{|c|c|c|c|c|c|c|}
\hline \multirow[t]{3}{*}{ Clinical best practice costs } & \multirow{3}{*}{$\begin{array}{l}\text { Median } \\
(\$)\end{array}$} & \multicolumn{2}{|c|}{ Confidence interval } & \multirow{3}{*}{$\begin{array}{l}\text { Median } \\
\text { (c) }\end{array}$} & \multicolumn{2}{|c|}{ Confidence interval } \\
\hline & & 2.5 & 97.5 & & 2.5 & 97.5 \\
\hline & & $\begin{array}{l}\text { Percentile } \\
(\$)\end{array}$ & $\begin{array}{l}\text { Percentile } \\
(\$)\end{array}$ & & $\begin{array}{l}\text { Percentile } \\
\text { (c) }\end{array}$ & $\begin{array}{l}\text { Percentile } \\
\text { (c) }\end{array}$ \\
\hline & $N=867$ & & & $N=1$ & & \\
\hline Hand hygiene total cost & 170.15 & 26.42 & 335.54 & 19.63 & 3.05 & 38.70 \\
\hline Human resource cost & 159.73 & 20.04 & 321.93 & 18.42 & 2.31 & 37.13 \\
\hline \multirow[t]{2}{*}{ Product cost } & 10.43 & 6.38 & 13.61 & 1.20 & 0.74 & 1.57 \\
\hline & $N=102$ & $(\$)$ & & $N=1$ & (c) & \\
\hline Hygiene and sanitation of surfaces total cost & 21.85 & 13.34 & 30.90 & 21.42 & 13.08 & 30.29 \\
\hline Human resource cost & 9.17 & 0.66 & 18.22 & 8.99 & 0.65 & 17.86 \\
\hline \multirow[t]{2}{*}{ Product cost } & 12.68 & 12.68 & 12.68 & 12.43 & 12.43 & 12.43 \\
\hline & $N=85$ & $(\$)$ & & $N=1$ & (c) & \\
\hline Cleaning of small equipment total cost & 21.48 & 6.64 & 40.38 & 25.27 & 7.81 & 47.51 \\
\hline Human resource cost & 21.48 & 6.64 & 40.38 & 25.27 & 7.81 & 47.51 \\
\hline Reusable materials * & & & & - & - & - \\
\hline \multirow[t]{2}{*}{ Products for disinfection* } & & & & - & - & - \\
\hline & $N=57$ & $(\$)$ & & $N=1$ & (c) & \\
\hline Additional precautions total cost & 235.33 & 26.62 & 462.76 & 412.86 & 31.32 & 544.42 \\
\hline \multirow[t]{2}{*}{ Human resource cost } & 235.33 & 26.62 & 462.76 & 412.86 & 31.32 & 544.42 \\
\hline & $N=720$ & $(\$)$ & & $N=1$ & (c) & \\
\hline Personal protective equipment (PPE) total cost & 546.76 & 445.04 & 660.67 & 75.94 & 61.81 & 91.76 \\
\hline Human resource cost & 114.10 & 12.38 & 228.01 & 15.85 & 1.72 & 31.67 \\
\hline PPE material & 432.66 & 432.66 & 432.66 & 60.09 & 60.09 & 60.09 \\
\hline \multirow[t]{2}{*}{ Screening* } & $N=3$ & - & - & - & - & - \\
\hline & $N=1831$ & $(\$)$ & & $N=1$ & (द) & \\
\hline $\begin{array}{l}\text { Total clinical best practice costs for two hours of } \\
\text { observation }\end{array}$ & 996.23 & 518.33 & $1,530.60$ & 54.41 & 28.31 & 83.59 \\
\hline Total clinical best practice costs per hour & 498.11 & 259.16 & 765.30 & 27.20 & 14.15 & 41.80 \\
\hline
\end{tabular}

Overall, for two hours of observation, the median cost for 867 actions of hand hygiene was $\$ 170.15$ (95\% Cl: 26.42-335.54), which represents 19.6 cents per action. For cleaning and disinfection of surfaces, the cost was $\$ 21.85$ (95\% Cl: 13.34-30.90) or 21.4 cents per action, while cleaning of small equipment (85 actions) was \$21.48 (95\% Cl: 6.64-40.38) for human resource costs, or 25.3 cents per action. Material costs were not captured in this category. Additional precautions median cost was $\$ 235.33$ (95\% Cl: 26.62-462.76) or \$4.13 per action. The 720 actions of donning or removing PPE median cost was $\$ 546.76$ (95\% Cl: $445.04-660.67)$ or 75.9 cents per action. Screening costs were not calculated as the number of samples taken for screening was too small $(n=3)$. Finally, the total median costs for the five categories of CBP assessed were $\$ 996.23$ for two hours ( $95 \%$ Cl: $518.33-$ 1530.60) of observation or $\$ 498.11$ per hour (95\% Cl: $259.16-765.30)$, which equalled 27.2 cents total median individual CBP action cost per hour. 


\section{Discussion}

In this pilot project of TDABC we assessed the costs of time, materials and products required to undertake essential clinical IPC practices by observing 48 healthcare workers in two Quebec hospitals. Our findings reveal that the costs of preventing the transmission of infection are remarkably low, even when the action is performed by the highest paid personnel (median cost being 27.2 cents per action). Weighed against the risk of infection and illness and subsequent monetary and human cost, this analysis supports the existing literature that describes the cost and cost-benefit of investing in resources that support compliance with IPC measures (14,31-33). This study also importantly provides an assessment of costs of infection prevention in a pre-pandemic context and thus serves as a baseline against which to compare future healthcare economic analyses.

When performed properly, hand hygiene is considered the single most important way to limit the spread of communicable diseases (34). Subjectively, healthcare personnel may consider the time spent on hand hygiene as adequate, however the times recorded in this study did not reach WHO standards (34). Indeed, in our observations, median time for hand washing ranged from 12.41 and 16.04 seconds across personnel, with a median cost of 19.6 cents per action. When using soap and water, the WHO suggests 40-60 seconds for an entire wash from the beginning to the end of the activity; we recorded median times of 22.16 seconds or $55 \%$ of the lower WHO limit. When using hydroalcoholic solutions that were wall or table mounted, median times were 13.95 and 13.83 seconds respectively, which also did not meet the WHO standard of 20-30 seconds minimum for rubbing using an alcoholbased formulation, although it came slightly closer at $69 \%$ of the lower limit. Compliant hand washing was observed among personnel that used pocket size gel, with a median time of 27.77 seconds which surpassed the minimum limit of 20 seconds by 7 seconds or $39 \%$. This may be due to the ease and efficiency of using a portable hand gel that allows for disinfecting one's hands during other tasks, such as talking with a patient, or traveling between rooms. Some evidence exists for installing dispensers close to their place of use to improve handwashing compliance (35). However, a recent systematic review outlines challenges with changing hand hygiene habits, and proposes that behaviour change is most successful if it is gradual and supported by leadership in a work environment that emphasizes the importance of patient safety $(36,37)$.

Environmental contamination is an established risk factor for developing a $\mathrm{HCAl}$, with patient rooms serving as a reservoir for multi drug resistant organisms that may infect new and susceptible patients $(38,39)$. We observed the cleaning of surfaces as carried out exclusively by hygiene and sanitation staff. The median time for disinfection was 541.53 seconds, or 9 minutes 2 seconds, with a total cost that included products used, of $\$ 21.85$, or 21.9 cents per action. A longer median time of 14 minutes 32 seconds was observed for terminal cleaning, which is done after patients are discharged or transferred. Enhanced patient room disinfection strategies, including those that target terminal cleaning (40) are essential as viruses such as the corona or influenza virus survive on dry surfaces for a few days, while bacteria such as Methicillin-resistant Staphylococcus aureus (MRSA) can persist for months (41). Despite this, few studies assess the costs of environmental cleaning interventions. One initiative, the Researching Effective Approaches to Cleaning in Hospitals (REACH) study, tested an environmental cleaning bundle in 11 Australian hospitals (33). Five components (product, cleaning technique, training of staff, audit and communication) were implemented with environmental services staff. Outcomes measured included: 1 ) the reductions of infections per month with the organisms MRSA and Vancomycin-resistant enterococci (VRE); 2) the thoroughness of hospital cleaning and; 3) an economic analysis of costs and savings. The bundle was successful in reducing infections and pathogen counts, and the implementation cost $\$ 349,000$ Australian dollars (AUD) but it generated $\$ 147,500$ in cost savings. Infections prevented from MRSA and VRE returned a conservatively estimated net monetary profit of $\$ 1.02$ million AUD. Costs were obtained for the change of type of disinfectant use, as well as for all human resources required to implement and receive training in the intervention, but neither regular human resource (time) nor product/material use were assessed. Considering the importance of hospital environmental cleaning, more micro costing analyses of human and product resource costs are required.

Although this pilot study took place prior to the beginning of the pandemic, the basic and additional precautions undertaken by staff were the CBPs with the highest calculated costs. The donning and removing of personal protective equipment (PPE) had a median total cost of $\$ 546.76$, or 75.9 cents per action. Twenty percent (20\%) of this ( 16 cents) was attributable to the time it took staff to put on gloves, gowns or masks, while the majority of the cost ( $80 \%$ or 60 cents) represented the materials used per action. For isolation measures, the median total costs were $\$ 235.33$ for two hours of observation, or $\$ 4.13$ per action. Over the course of a regular shift of eight hours this would represent a total median cost of $\$ 941.32$. However, during the study period, no observations of airborne or airborne-contact precautions occurred.

In the current state of the COVID-19 pandemic, PPE is increasingly used for the treatment of all patients, and new equipment such as eye protection (face shield or goggles) is becoming standard practice. This increased use parallels increased costs, as was seen during the Middle East Respiratory Syndrome (MERS-CoV) epidemic. In one hospital in Saudi Arabia with 17 positive cases of MERS, the use of surgical masks increased 5-fold and the use of $\mathrm{N} 95$ masks increased 10-fold per 1,000 patient days (42). In the three month period studied, allowing also for the increase in compliance of hand hygiene, this resulted in a $\$ 16,400$ per month increase in IPC costs. During this past year, this same driving force in the supply chain, coupled with demand in the general public for PPE, has caused the market demand to explode, resulting in global shortages and price increases (43). In March of 2020, the WHO reported that prices of surgical masks had already increased six fold, N95 respirators had tripled, and surgical gown prices had doubled (44). Our study contributes new knowledge related to pre-COVID-19 costs which will allow researchers to compare future PPE use and cost increases.

Due to the small number $(n=3)$ of screening tests done during the one-month study period we were unable to calculate the costs of human or product resources for infectious disease screening. Future work planned by our team will allow for a one-year time frame from which to collect data from medical records. This will provide accurate infectious disease screening tests performed without the need for observation.

Overall, the time motion observations using our guide were feasible and acceptable to both the observers and staff being observed. We ensured that the same observer followed a staff member during the course of the study for two hours and 10 minutes each day. The initial 10 minutes was not

Page $10 / 15$ 
measured, but allowed the observer to adjust to the tasks and pace being set by the staff member being observed. These procedures allowed for some dissipation of the Hawthorne effect, a known confounder in observational studies of healthcare practices such as hand washing (45). The micro-costing data collected in this study were captured with an observational prospective study design, the optimal technique for obtaining accurate cost estimates to inform resource allocation decisions (24).

Our study has several limitations. We were unable to capture two category costs: those of screening and the costs of materials used for the cleaning of small equipment (missing data). We did not include doctors in this pilot project; their higher wages would augment the average human resource cost across the average costs presented here. To address these limitations and to test the reproducibility of these results, we are undertaking a larger scale study in a context that has been influenced by COVID-19, using the same time motion guide.

\section{Conclusions}

The cost data retrieved from this study should be of great interest to policy makers, as even by conservative estimates the cost of the CBPs assessed were very low, from 20 cents to $\$ 4.13$ per action. Our results are relevant not only to stakeholders in Quebec's healthcare system but in other provinces and countries as well, as they provide arguments with which to make evidence-based decisions of resource allocation that affect the quality and safety of patient care. In this newly heightened context of COVID-19 risk of contagion, research in IPC is increasingly important to prioritize (46). There is an urgent need to understand that IPC programs are cost-effective. These programs must be acceptable to public officials, administrators, patients, and increasingly, the healthcare workers that are in contact with pathogens on a daily basis. If not, compliance with IPC measures will be low. As well, investments should also demonstrate a cost-benefit, such that the long term repercussions of patient safety are considered. This information will ultimately be useful for the care of the general population.

\section{Abbreviations}

BD

Bruno Dubreuil

CBP

Clinical best practice

CDC

Centers for Disease Control

CISSS

Integrated centre for health and social services

CIUSSS

Integrated university centre for health and social services

COVID-19

Corona Virus 2019

$\mathrm{CL}$

Catherine Larouche

DS

Drissa Sia

ET

Eric Tchouaket

HCAl

Healthcare Associated Infection

JL

Josiane Létourneau

$\mathrm{KK}$

Kelley Kilpatrick

NP

Natasha Parisien

SB

Sandra Boivin

SR

Stephanie Robins

WHO

World Health Organization

\section{Declarations}

\section{Ethics approval and consent to participate}


Participants provided written informed consent to participate. The research ethics board of the institutions were recruitment took place provided approval - study \# MP-28-2018-002).

\section{Consent for publication}

Not applicable

\section{Availability of data and materials}

The datasets used and/or analysed during the current study are available from the corresponding author on reasonable request.

\section{Competing interests}

The authors declare that they have no competing interests.

\section{Funding}

This study was funded by Fonds de Recherche du Québec-Santé (FRQS, http://www.frqs.gouv.qc.ca/en/) from April 1, 2017, to March 31, 2021, grant number \#35124 and the Canadian Institutes of Health Research (CIHR, https://cihr-irsc.gc.ca/e/193.html) grant \#409071.

Authors' contributions: ET, SR, SB, DS, KK, JL, BD, CL and NP and made substantial contributions to study conception and design. ET completed all the statistical analyses. All authors were involved in drafting and making revisions to critical intellectual content in the manuscript. All authors gave their final approval of the version to be published.

Acknowledgments: The authors are grateful to the Fonds de Recherche en Santé (FRQS) and for their financial support. The authors would like to thank Patrick Finken for his assistance with the web-based mobile platform for observations, the staff and managers at the public health department of the Laurentian Region and the nursing department of the Saguenay-Lac-Saint-Jean region.

\section{References}

1. Centres for Disease Control and Prevention. Healthcare Asssociated Infections: U.S. Department of Health \& Human Services; 2014 Available from: https://www.cdc.gov/hai/infectiontypes.html.

2. Saleem Z, Godman B, Hassali MA, Hashmi FK, Azhar F, Rehman IU. Point prevalence surveys of health-care-associated infections: a systematic review. Pathog Glob Health. 2019;113(4):191-205.

3. Russo PL, Stewardson AJ, Cheng AC, Bucknall T, Mitchell BG. The prevalence of healthcare associated infections among adult inpatients at nineteen large Australian acute-care public hospitals: a point prevalence survey. Antimicrobial resistance infection control. 2019;8:114.

4. Umscheid CA, Mitchell MD, Doshi JA, Agarwal R, Williams K, Brennan PJ. Estimating the proportion of healthcare-associated infections that are reasonably preventable and the related mortality and costs. Infect Control Hosp Epidemiol. 2011;32(2):101-14.

5. Cassini A, Plachouras D, Eckmanns T, Abu Sin M, Blank HP, Ducomble T, et al. Burden of Six Healthcare-Associated Infections on European Population Health: Estimating Incidence-Based Disability-Adjusted Life Years through a Population Prevalence-Based Modelling Study. PLoS Med. 2016;13(10):e1002150.

6. Arefian H, Hagel S, Heublein S, Rissner F, Scherag A, Brunkhorst FM, et al. Extra length of stay and costs because of health care-associated infections at a German university hospital. Am J Infect Control. 2016;44(2):160-6.

7. Serra-Burriel M, Keys M, Campillo-Artero C, Agodi A, Barchitta M, Gikas A, et al. Impact of multi-drug resistant bacteria on economic and clinical outcomes of healthcare-associated infections in adults: Systematic review and meta-analysis. PLOS ONE. 2020;15(1):e0227139.

8. Resar R, Griffin FA, Haraden C, Nolan TW. Using care bundles to improve health care quality. HI Innovation Series white paper. 2012;Cambridge, MA: Institute for Healthcare Improvement.

9. Canadian Patient Safety Institute. Infection Prevention and Control. 2021.

10. Government of Canada. Public Health Agency of Canada. Healthcare Acquired Infections. 2019.

11. Centers for Disease Control and Prevention. Guidelines for Environmental Infection Control in Health-Care Facilities, Recommendations of CDC and the Healthcare. Infection Control Practices Advisory Committee (HICPAC). 2003; 52(RR 10):[1-42 pp.]. Available from:

https://www.cdc.gov/mmwr/preview/mmwrhtml/rr5210a1.htm.

12. Liang T, First Affiliated Hospital of Zhejiang University. Handbook of COVID-19 Prevention and Treatment 2020.

13. Centers for Disease Control and Prevention. Comprehensive Hospital Preparedness Checklist for Coronavirus Disease 2019 (COVID-19)2020. Available from: https://www.cdc.gov/coronavirus/2019-ncov/downloads/HCW_Checklist_508.pdf.

14. Tchouaket E, Beogo I, Sia D, Kilpatrick K, Séguin C, Baillot A, et al. Economic analysis of healthcare-associated infection prevention and control interventions in medical and surgical units: systematic review using a discounting approach. J Hosp Infect. 2020;106(1):134-54.

15. Boev C, Kiss E. Hospital-Acquired Infections: Current Trends and Prevention. Crit Care Nurs Clin North Am. 2017;29(1):51-65.

16. Canadian Institute for Health Information. National Health Expenditure Trends, 1975-2019. Ottawa, ON 2019. 
17. Ministère des Finances du Québec. Budget 2018-2019: Plan économique du Québec- Des services de santé accessibles et de qualité 2018. Gouvernement du Québec; 2018.

18. Farbman L, Avni T, Rubinovitch B, Leibovici L, Paul M. Cost-benefit of infection control interventions targeting methicillin-resistant Staphylococcus aureus in hospitals: systematic review. Clin Microbiol Infect. 2013;19(12):E582-93.

19. Kaplan RS, Anderson SR. Time-driven activity-based costing. Harvard Business Review. 2004.

20. Keel G, Savage C, Rafiq M, Mazzocato P. Time-driven activity-based costing in health care: A systematic review of the literature. Health Policy. 2017;121(7):755-63.

21. Teisberg E, Wallace S, O'Hara S. Defining and Implementing Value-Based Health Care: A Strategic Framework. Acad Med. 2020;95(5):682-5.

22. Tchouaket E, Boivin S, Robins S, Sia D, Kilpatrick K, Brousseau S, et al. Development and validation of a time and motion guide to assess the costs of prevention and control interventions for nosocomial infections: A Delphi method among experts. PLOS ONE. 2020;15(11):e0242212.

23. Tan SS, Rutten FF, van Ineveld BM, Redekop WK, Hakkaart-van Roijen L. Comparing methodologies for the cost estimation of hospital services. The European journal of health economics: HEPAC: health economics in prevention care. 2009;10(1):39-45.

24. Chapel JM, Wang G. Understanding cost data collection tools to improve economic evaluations of health interventions. Stroke Vasc Neurol. 2019;4(4):214-22.

25. Lopetegui M, Yen PY, Lai A, Jeffries J, Embi P, Payne P. Time motion studies in healthcare: what are we talking about? J Biomed Inform. 2014;49:292-9.

26. Comité patronal de négociation du secteur de la santé et des services sociaux. Nomenclature en vigeur. Ministère de la Santé et des Services sociaux; 2020.

27. Ministère de l'Économie et de l'Innovation. Les marchés publics québécois. 2021.

28. da Silva Etges APB, Cruz LN, Notti RK, Neyeloff JL, Schlatter RP, Astigarraga CC, et al. An 8-step framework for implementing time-driven activitybased costing in healthcare studies. The European journal of health economics: HEPAC: health economics in prevention care. 2019;20(8):1133-45.

29. Kaplan RS, Porter ME. The Big Idea: How to Solve the Cost Crisis in Health Care. Harvard Business Review; 2011.

30. Polit D, Beck CT. Nursing Research: Generating and Assessing Evidence for Nursing Practice: 11th edition. Wolters Kluwer Law \& Business 2020.

31. Mac S, Fitzpatrick T, Johnstone J, Sander B. Vancomycin-resistant enterococci (VRE) screening and isolation in the general medicine ward: a costeffectiveness analysis. Antimicrobial Resistance Infection Control. 2019;8(1):168.

32. Dick AW, Perencevich EN, Pogorzelska-Maziarz M, Zwanziger J, Larson EL, Stone PW. A decade of investment in infection prevention: a costeffectiveness analysis. Am J Infect Control. 2015;43(1):4-9.

33. White NM, Barnett AG, Hall L, Mitchell BG, Farrington A, Halton K, et al. Cost-effectiveness of an Environmental Cleaning Bundle for Reducing Healthcare-associated Infections. Clinical infectious diseases: an official publication of the Infectious Diseases Society of America. 2020;70(12):2461-8.

34. World Health Organization. WHO Guidelines on hand hygiene in health care, first global patient safety challenge clean care is safer care2009. Available from: https://apps.who.int/iris/bitstream/handle/10665/44102/9789241597906_eng.pdf.

35. Gould DJ, Moralejo D, Drey N, Chudleigh JH, Taljaard M. Interventions to improve hand hygiene compliance in patient care. Cochrane Database of Systematic Reviews. 2017(9).

36. Martos-Cabrera MB, Mota-Romero E, Martos-García R, Gómez-Urquiza JL, Suleiman-Martos N, Albendín-García L, et al. Hand Hygiene Teaching Strategies among Nursing Staff: A Systematic Review. Int J Environ Res Public Health. 2019;16(17):3039.

37. Létourneau J, Alderson M, Leibing A. Positive deviance and hand hygiene of nurses in a Quebec hospital: What can we learn from the best? Am J Infect Control. 2018;46(5):558-63.

38. Cohen B, Liu J, Cohen AR, Larson E. Association Between Healthcare-Associated Infection and Exposure to Hospital Roommates and Previous Bed Occupants with the Same Organism. Infect Control Hosp Epidemiol. 2018;39(5):541-6.

39. Weber DJ, Anderson D, Rutala WA. The role of the surface environment in healthcare-associated infections. Curr Opin Infect Dis. 2013;26(4):33844.

40. Anderson DJ, Chen LF, Weber DJ, Moehring RW, Lewis SS, Triplett PF, et al. Enhanced terminal room disinfection and acquisition and infection caused by multidrug-resistant organisms and Clostridium difficile (the Benefits of Enhanced Terminal Room Disinfection study): a clusterrandomised, multicentre, crossover study. Lancet. 2017;389(10071):805-14.

41. Kramer A, Schwebke I, Kampf G. How long do nosocomial pathogens persist on inanimate surfaces? A systematic review. BMC Infect Dis. 2006;6(1):130.

42. Al-Tawfiq JA, Abdrabalnabi R, Taher A, Mathew S, Rahman KA. Infection control influence of Middle East respiratory syndrome coronavirus: A hospital-based analysis. Am J Infect Control. 2019;47(4):431-4.

43. Cohen J, Rodgers YVM. Contributing factors to personal protective equipment shortages during the COVID-19 pandemic. Preventive medicine. 2020;141:106263.

44. World Health Organization. Shortage of personal protective equipment endangering health workers worldwide2020. Available from: https://www.who.int/news/item/03-03-2020-shortage-of-personal-protective-equipment-endangering-health-workers-worldwide.

Page $13 / 15$ 
45. Purssell E, Drey N, Chudleigh J, Creedon S, Gould DJ. The Hawthorne effect on adherence to hand hygiene in patient care. J Hosp Infect. 2020;106(2):311-7.

46. Dancer SJ. Covid-19 exposes the gaps in infection prevention and control. Infection disease health. 2020;25(4):223-6.

\section{Figures}

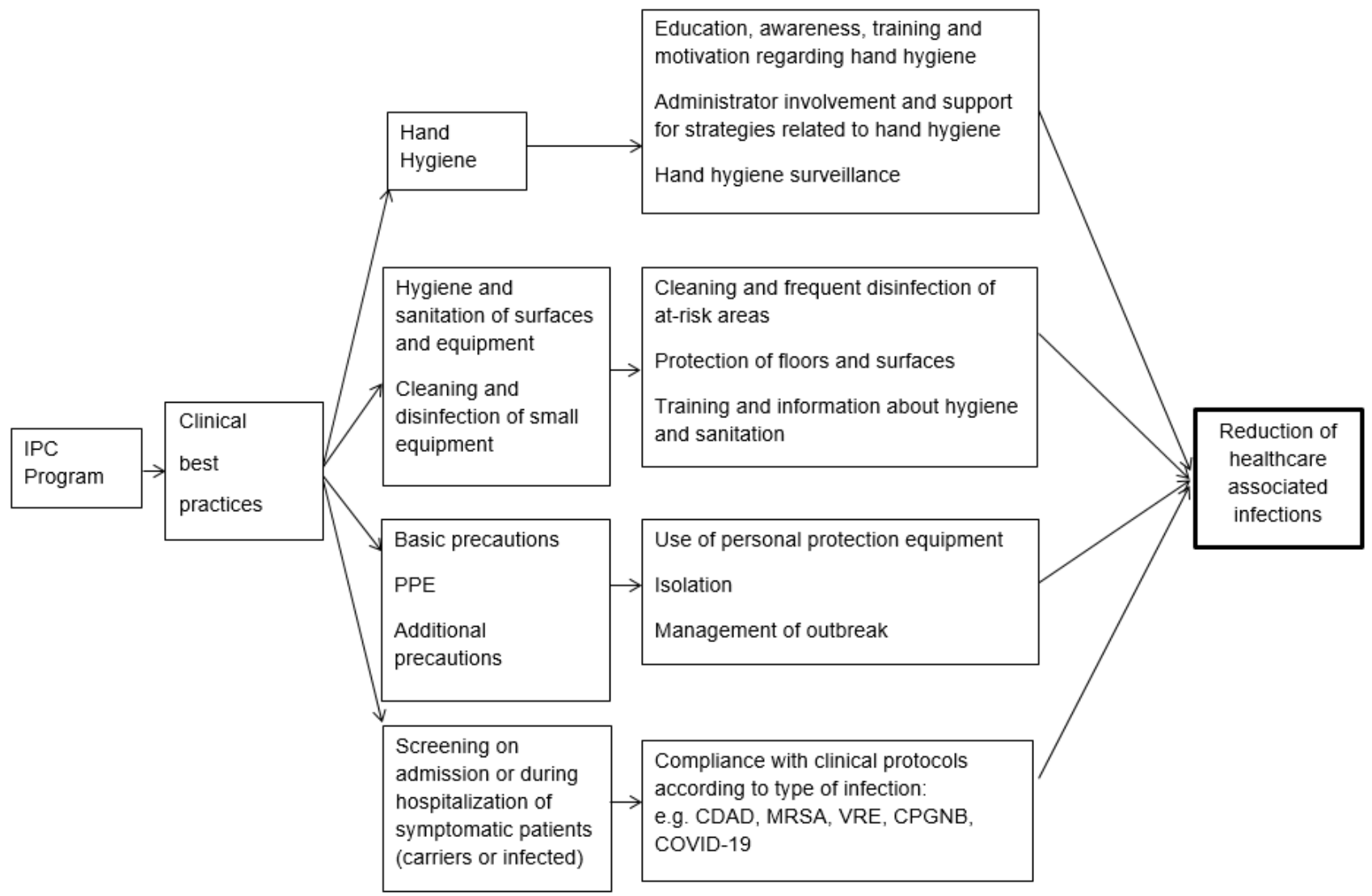

Figure 1

Infection prevention and control clinical best practices 


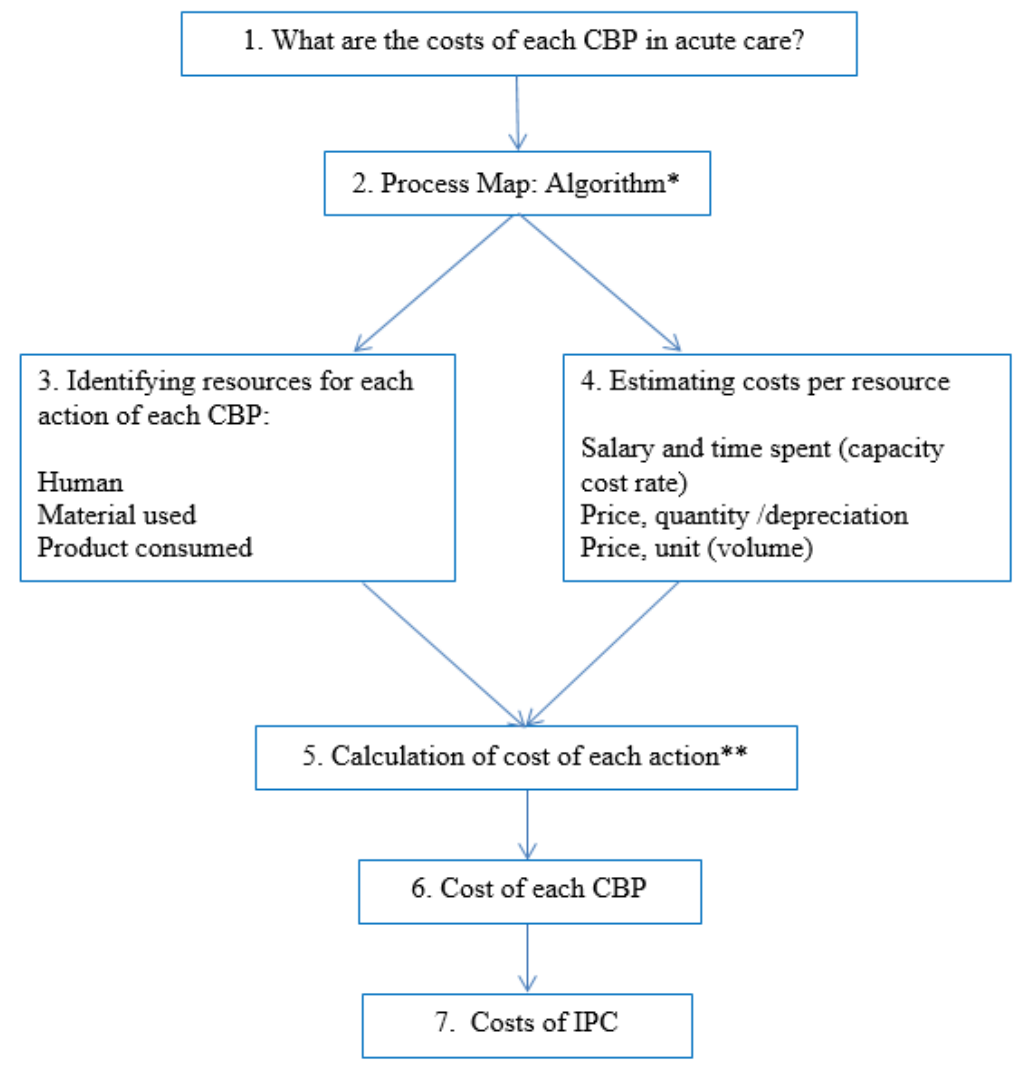

\section{Figure 2}

Costing Procedure Framework Legend: *See Figure $1{ }^{\star \star}$ Cost calculations are described in Table 1.

\section{Supplementary Files}

This is a list of supplementary files associated with this preprint. Click to download.

- Supplementaryfile1.Algorithm.docx

- Supplementaryfile2.TimeMotionGuide.docx 